\title{
INFLUÊNCIA DA TEMPERATURA DE AQUECIMENTO NA RESISTÊNCIA MECÂNICA DE UM AÇO MICROLIGADO *
}

\author{
Pedro Gabriel Bonella de Oliveira ${ }^{1}$ \\ Rosana Vilarim da Silva ${ }^{2}$ \\ Bruna Helena Malovini Loiola ${ }^{3}$ \\ André Itman Filho ${ }^{4}$
}

\section{Resumo}

O processo usual para elaboração de aços utilizados na indústria de petróleo e gás é a laminação controlada, porém, não é adequado para fabricação de peças de geometria complexa, como juntas e conexões. Nestes casos, o forjamento é a melhor opção, pois permite maior diversidade de conformação. A produção de componentes por esta técnica exige tratamentos de têmpera e revenimento para obtenção das propriedades exigidas em trabalho. O objetivo deste projeto é avaliar o comportamento em tração e a microestrutura de um aço microligado submetido ao aquecimento a 855,900 e $1000^{\circ} \mathrm{C}$. Modelos termodinâmicos de Kasatkin foram utilizados para cálculo das temperaturas de transformação austenítica. Os resultados mostram que o tratamento realizado na zona de transformação intercrítica favorece a formação de uma estrutura bifásica de ferrita e martensita com maiores valores da tensão de escoamento e resistência máxima à tração. Nos tratamentos térmicos realizados em maiores temperaturas há diminuição na resistência mecânica, porém com aumento no alongamento em consequência de uma estrutura composta de bainita e ferrita acicular.

Palavras-chave: Aços microligados; Forjamento; Tratamentos térmicos; Propriedades mecânicas.

\section{EFFECT OF HEATING TEMPERATURE ON THE MECHANICAL PROPERTIES OF A FORGED MICROALLOYED STEEL}

\section{Abstract}

Controlled rolling is the most usual way for fabrication of steels used in the oil and gas industry, however, this process is unsuitable to produce complex geometry tools, such as joints and connections. Forging presents as a viable option to manufacture this kind of components, allowing a wider diversity of conformation. Fabrication by forging demands the use of quenching and tempering treatments to achieve the work required properties. The objective of this project is to evaluate the strain behavior and microstructure of a microalloyed steel under heating at 855,900 and $1000{ }^{\circ} \mathrm{C}$. Kasatkin thermodynamic models were used to calculate the austenitic transformation temperatures. Results show that the treatment in the intercritical region promotes a bifasic microstructure of ferrite and martensite with higher values of yield strenght and ultimate stress. In higher temperatures treatments there is reduction in the mechanical properties with higher alongation due to a structure composed of bainite and acicular ferrite.

Keywords: Microalloyed steels; Forging; Heat treatments; Mechanical properties.

1 Engenheiro Metalurgista e Mestrando em Engenharia de Metalurgia e Materiais. PROPEMM Instituto Federal do Espírito Santo, Vitória, ES, Brasil.

2 Doutora em Ciência e Engenharia dos Materiais. PPGTECS - Instituto Federal do Espírito Santo, Vitória, ES, Brasil.

3 Graduanda em Engenharia Metalúrgica. Instituto Federal do Espírito Santo, Vitória, ES, Brasil.

4 Doutor em Ciência e Engenharia dos Materiais. PROPEMM - Instituto Federal do Espírito Santo, Vitória, ES, Brasil. 


\section{INTRODUÇÃO}

Os aços microligados são utilizados nas indústrias de extração de petróleo e gás, construção, transporte e representam aproximadamente $12 \%$ da produção mundial [1]. Nesta classe se destacam os aços de alta resistência e baixa liga (ARBL), que são caracterizados por conter quantidades bem pequenas de elementos formadores de carbonetos, responsáveis pelo endurecimento secundário, refino de grão e transformações de fase.

Na fabricação de dutos para a indústria de petróleo e gás, estes aços devem seguir as especificações da norma API (American Petroleum Institute) $5 \mathrm{~L}$. O processo mais utilizado para fabricação dos aços API é a laminação controlada, que associada aos tratamentos térmicos proporciona excelente resistência mecânica, tenacidade e soldabilidade.

Com relação aos acessórios utilizados na construção de dutos tais como flanges, conexões e centralizadores de tubos, a laminação não é uma técnica adequada e nestes casos, para fabricar componentes com maior complexidade geométrica, o processo de forjamento é uma opção mais viável.

Este processo, muito utilizado na indústria automotiva, permite uma ampla faixa de dimensões e formas, com controle da microestrutura e propriedades mecânicas por meio de tratamentos térmicos de têmpera e revenimento.

Nos aços ARBL com menor teor de carbono, uma alternativa para aumentar as propriedades mecânicas é o resfriamento rápido, a partir da região intercrítica entre a temperatura austenítica inferior $\left(A c_{1}\right)$ e superior $\left(A c_{3}\right)$, com a formação de martensita [2].

Associado ao processo de fabricação, a utilização de nióbio, titânio e vanádio é importante no desenvolvimento de aços de alta resistência. É conhecido o efeito destes elementos para promover o endurecimento por solução sólida e o refino de grão, que favorecem o aumento da resistência mecânica dos aços [3]. Outro elemento de liga importante é o molibdênio que favorece a formação de produtos de transformação displacivos com aumento da resistência mecânica e da tenacidade [4]. Desta forma e considerando a composição química e diferentes tratamentos térmicos, o objetivo deste trabalho foi avaliar a microestrutura e as propriedades mecânicas em tração de um aço microligado forjado submetido ao aquecimento a 855,900 e $1000{ }^{\circ} \mathrm{C}$ com posterior resfriamento resfriado em água.

\section{MATERIAIS E MÉTODOS}

O aço microligado foi elaborado em um forno de indução com capacidade para 300 $\mathrm{kg}$ na fundição Grupo Metal em Tietê/SP. O metal líquido foi vazado em uma lingoteira de ferro fundido e posteriormente forjado na forma de barras com secção quadrada. Os teores dos elementos químicos foram determinados por meio do espectrômetro de emissão ótica da marca Oxford Instruments, modelo FoundryMaster Pro.

O lingote obtido após o vazamento foi cortado em três partes com pesos similares, aquecido a $1050{ }^{\circ} \mathrm{C}$ e forjado em três barras com secção quadrada de aproximadamente $38 \mathrm{~mm}$ de aresta e $1500 \mathrm{~mm}$ de comprimento. Os últimos passes foram realizados com a temperatura mínima de $850{ }^{\circ} \mathrm{C}$ para refinar o grão. Posteriormente, as barras foram fresadas com $30 \mathrm{~mm}$ de lado. Por fim, foi realizado o reaquecimento das barras a $930{ }^{\circ} \mathrm{C}$ por duas horas, seguido de resfriamento em 
forno, para homogeneizar a microestrutura. O processamento termomecânico dos aços é mostrado na Figura 1.

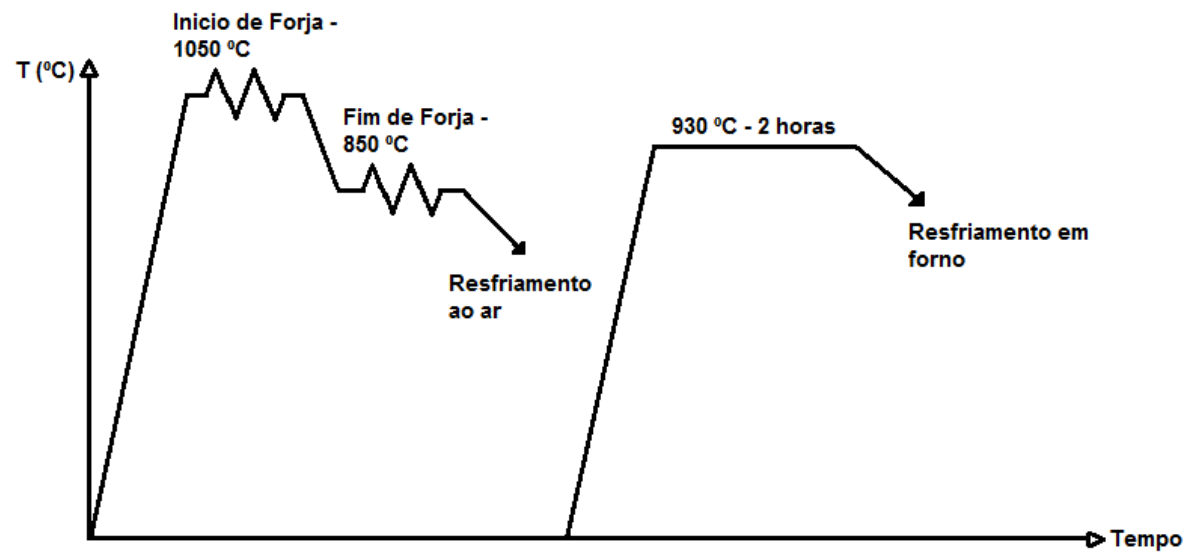

Figura 1. Desenho esquemático do processamento termomecânico das barras do aço microligado.

Amostras na orientação longitudinal com $15 \times 15 \times 100 \mathrm{~mm}$ foram retiradas para análises microestruturais e posterior usinagem dos corpos de prova. As temperaturas de transformação austenítica foram determinadas por meio das equações propostas por Kasatkin [5]:

$$
\begin{aligned}
& \mathrm{Ac}_{1}=723-7,08 \mathrm{Mn}+37,7 \mathrm{Si}+18,1 \mathrm{Cr}+44,2 \mathrm{Mo}+8,95 \mathrm{Ni}+50,1 \mathrm{~V}+21,7 \mathrm{Al}+3,18 \mathrm{~W} \\
& \text { + } 297 \mathrm{~S}-830 \mathrm{~N} \text { - 11,5C.Si - 14Mn.Si - 3,1Si.Cr - 57,9C.Mo - 15,5Mn.Mo - 5,28C.Ni - }
\end{aligned}
$$

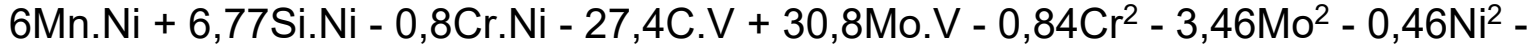

$$
\begin{aligned}
& 28 \mathrm{~V}^{2} \\
& A c_{3}=912-370 \mathrm{C}-27,4 \mathrm{Mn}+27,3 \mathrm{Si}-6,35 \mathrm{Cr}-32,7 \mathrm{Ni}+95,2 \mathrm{~V}+190 \mathrm{Ti}+72 \mathrm{Al}+ \\
& 64,5 \mathrm{Nb}+5,57 \mathrm{~W}+332 \mathrm{~S}+276 \mathrm{P}+485 \mathrm{~N}-900 \mathrm{~B}+16,2 \mathrm{C} \cdot \mathrm{Mn}+32,3 \mathrm{C} . \mathrm{Si}+15,4 \mathrm{C} \cdot \mathrm{Cr}+ \\
& \text { 48C.Ni + 4,32Si.Cr - 17,3Si.Mo - 18,6Si.Ni + 4,8Mn.Ni + 40,5Mo.V + 174C } \mathrm{C}^{2}+ \\
& 2,46 \mathrm{Mn}^{2}-6,86 \mathrm{Si}^{2}+0,322 \mathrm{Cr}^{2}+9,9 \mathrm{Mo}^{2} 1,24 \mathrm{Ni}^{2}-60,2 \mathrm{~V}^{2}
\end{aligned}
$$

O tratamento térmico de têmpera foi realizado em um forno tipo mufla. As amostras foram mantidas nas temperaturas de 855,900 e $1000^{\circ} \mathrm{C}$ por 30 minutos e resfriadas em água. Após preparação metalográfica convencional as amostras foram submetidas ao ataque químico com o reagente Nital $2 \%$ e tempo de aplicação de aproximadamente 30 segundos. As imagens obtidas foram capturadas no Microscópio Ótico Confocal (MOC) modelo DCM 3D Leica. Os ensaios de tração foram realizados em uma máquina de ensaios universal modelo Emic DL 10000 de acordo com a norma ASTM E8/E8M.

\section{RESULTADOS E DISCUSSÃO}

A composição química do aço microligado está apresentada na Tabela 1.

Tabela 1. Composição química do aço microligado (\% em peso).

\begin{tabular}{cccccccccccc}
\hline $\mathbf{C}$ & $\mathbf{S i}$ & $\mathbf{M n}$ & $\mathbf{P}$ & $\mathbf{S}$ & $\mathbf{C r}$ & $\mathbf{M o}$ & $\mathbf{N}$ & $\mathbf{N i}$ & $\mathbf{N b}$ & $\mathbf{T i}$ & $\mathbf{V}$ \\
\hline 0,11 & 0,09 & 0,33 & 0,03 & 0,02 & 0,37 & 0,05 & 0,006 & 0,4 & 0,08 & 0,01 & 0,01 \\
\hline
\end{tabular}


Os valores das temperaturas de transformação austenítica foram $736{ }^{\circ} \mathrm{C}$ para $\mathrm{Ac}_{1} \mathrm{e}$ $881^{\circ} \mathrm{C}$ para $\mathrm{Ac}_{3}$. Segundo o modelo de Kasatkin, o aquecimento realizado a $855^{\circ} \mathrm{C}$ foi na zona intercrítica e os demais na região austenítica.

A análise microestrutural revela que o resfriamento após o aquecimento na temperatura de $855^{\circ} \mathrm{C}$ promove a formação de uma estrutura bifásica de ferrita poligonal (FP) e martensita (M), como se observa na Figura 2. O resfriamento a partir da zona intercrítica favorece a formação de martensita, devido ao maior teor de carbono na austenita nesta região.

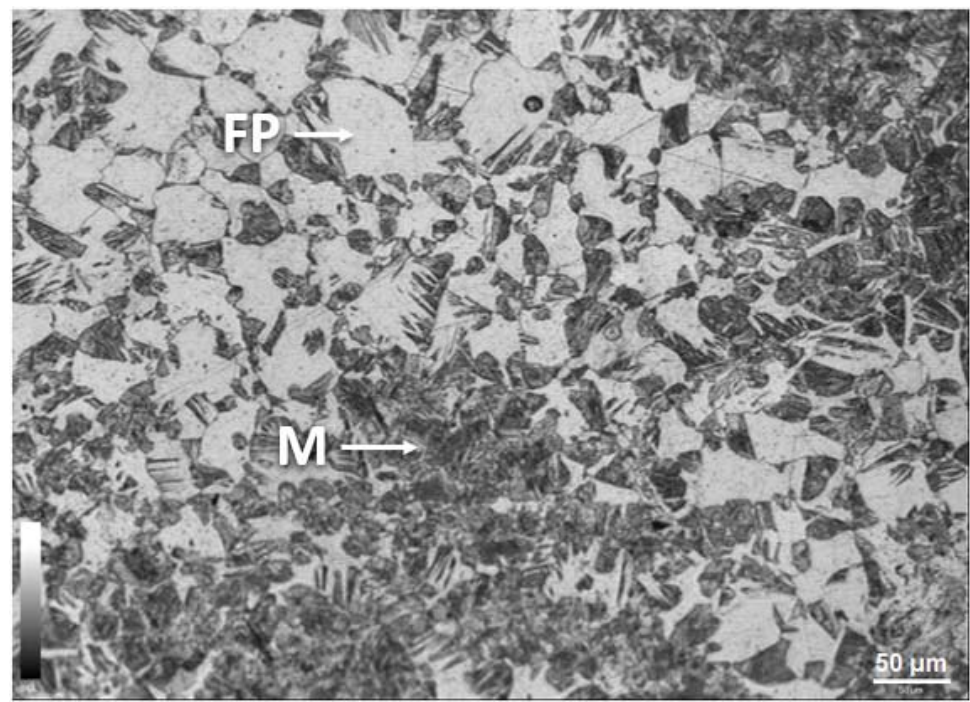

Figura 2. Aço aquecido a $855^{\circ} \mathrm{C}$ e resfriado em água.

Os tratamentos realizados acima da temperatura crítica favorecem a formação dos produtos típicos dos aços baixo carbono submetidos ao resfriamento brusco. Neste caso, o material é aquecido até a completa austenitização e com o resfriamento realizado fora das condições de equilíbrio são formadas estruturas displacivas. $\mathrm{Na}$ Figura 3, resfriamento a partir de $900^{\circ} \mathrm{C}$, é possível observar uma estrutura mista de ferrita acicular (AcF), bainita (B), ferrita alotriomórfica nos contornos de grão (FA) e também ferrita de Widmanstatten (WF).

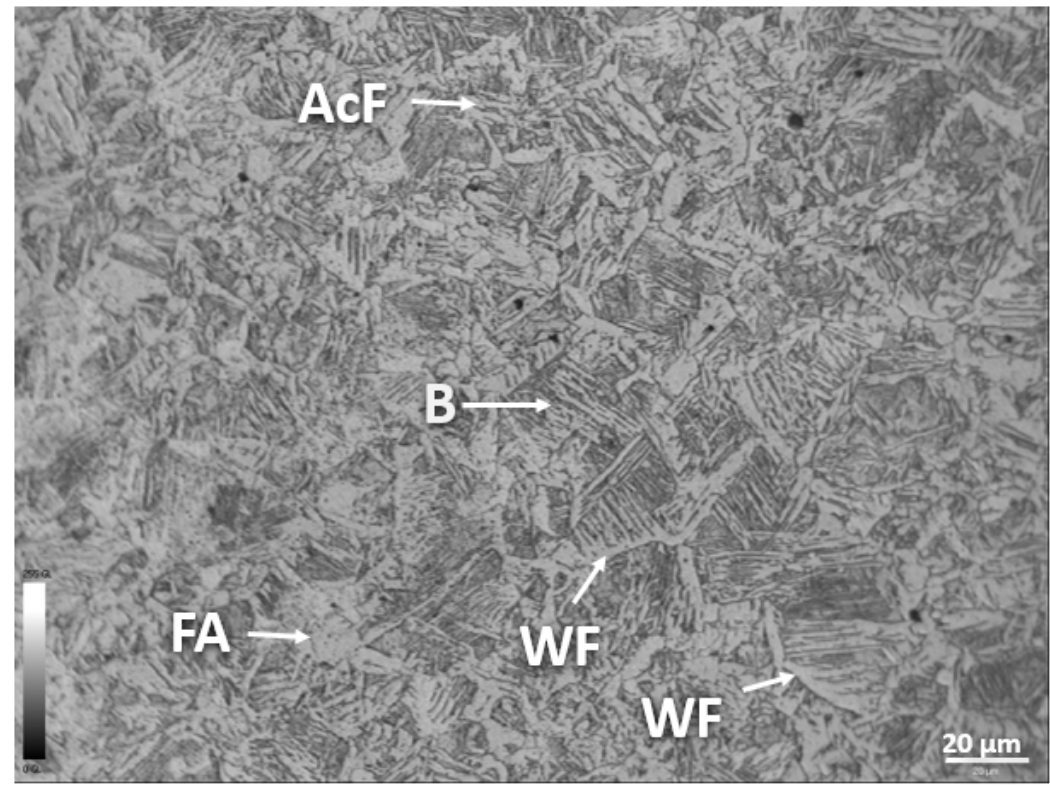

Figura 3. Aço aquecido a $900{ }^{\circ} \mathrm{C}$ e resfriado em água. 
Nas amostras aquecidas a $1000{ }^{\circ} \mathrm{C}$ a formação de ferrita Widmanstatten e alotriomórfica é menor e a microestrutura resultante é praticamente de ferrita acicular e bainita (Figura 4).

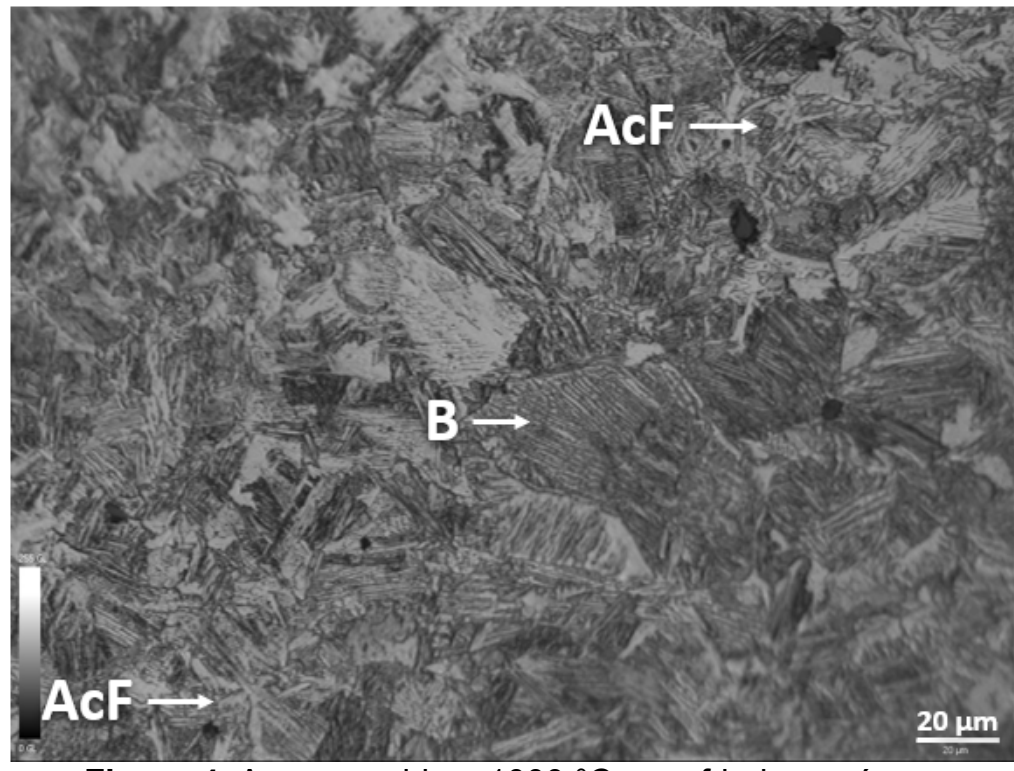

Figura 4. Aço aquecido a $1000^{\circ} \mathrm{C}$ e resfriado em água.

A Tabela 2 apresenta os resultados dos ensaios de tração nas diferentes condições de tratamento térmico.

Tabela 2. Valores da tensão de escoamento, resistência à tração e alongamento nas diferentes temperaturas de aquecimento.

\begin{tabular}{cccc}
\hline Temperatura $\left({ }^{\circ} \mathbf{C}\right)$ & $\boldsymbol{\sigma}_{\mathrm{e}}(\mathbf{M P a})$ & $\boldsymbol{\sigma}_{\max }(\mathrm{MPa})$ & Al. (\%) \\
\hline 855 & $833 \pm 11$ & $1071 \pm 10$ & $12,5 \pm 0,4$ \\
\hline 900 & $537 \pm 07$ & $724 \pm 09$ & $14,8 \pm 0,6$ \\
\hline 1000 & $425 \pm 06$ & $617 \pm 08$ & $21,7 \pm 0,9$ \\
\hline
\end{tabular}

No aquecimento a $855^{\circ} \mathrm{C}$ os valores de $\sigma_{e}$ e $\sigma_{\max }$ foram superiores com relação aos das temperaturas mais altas. Isto ocorreu pela formação da fase martensítica. No entanto, esta fase é responsável pelos menores valores de alongamento. Nas temperaturas de 900 e $1000{ }^{\circ} \mathrm{C}$ houve a redução em $\sigma_{\mathrm{e}}$ e $\sigma_{\max }$, uma vez que os constituintes formados, ferrita acicular e bainita, apresentam resistência mecânica inferior à da martensita. A presença de ferrita de Widmanstatten pode ser a responsável pelo menor valor de alongamento exibido no aquecimento a $900{ }^{\circ} \mathrm{C}$. Esta fase associada à presença de ferrita alotriomórfica nos contornos de grão, bem como o espaçamento e a orientação das placas na matriz, pode ser prejudicial à tenacidade do material [6].

Com relação ao aumento do alongamento no aquecimento a $1000{ }^{\circ} \mathrm{C}$, o resultado pode ser explicado pela maior quantidade de ferrita acicular no aço após análise qualitativa de várias amostras. A nucleação intergranular das placas de ferrita nas inclusões não metálicas e o crescimento em várias orientações, diferente do paralelismo da bainita e da ferrita de Widmanstatten, é responsável pela dificuldade de propagação das trincas no material [7]. Esta fase é considerada a ideal nos aços utilizados na indústria de óleo e gás em razão da excelente combinação de resistência mecânica e tenacidade. 


\section{CONCLUSÃO}

- Os cálculos das temperaturas de transformação austenítica indicam que os aquecimentos a 900 e $1000{ }^{\circ} \mathrm{C}$ são realizados na região austenítica, enquanto que na temperatura de $855^{\circ} \mathrm{C}$ ocorre em uma zona intercrítica;

- O tratamento na zona intercrítica é responsável pela formação de uma estrutura bifásica de ferrita e martensita no material;

- O aço tratado na zona intercrítica apresenta resistência mecânica superior aos demais, porém com menor ductilidade;

- O aço tratado a $1000{ }^{\circ} \mathrm{C}$ apresentou melhor combinação de resistência e alongamento devido à estrutura predominante de ferrita acicular e bainita.

\section{Agradecimentos}

Os autores agradecem à Fapes pelo apoio financeiro, à Fundição Grupo Metal pelas amostras do aço forjado, à Finep e à CAPES pela bolsa de mestrado.

\section{REFERÊNCIAS}

1 Kumar, B. V. R. A review on importance of microalloying in steel. International Journal of Mechanical Engineering and Technology (IJMET). 2014; 5:p.187-193.

2 Kang, J.; Wang, C.; Wang G. D. Microstructural characteristics and impact fracture behavior of a high-strength low-alloy steel treated by intercritical heat treatment. Materials Science and Engineering A. 2012; 553: p.96-104.

3 Gladman, T. The Physical Metallurgy of Microalloyed Steels. Leeds: Maney Pub. 1997.

4 Isasti, N. et al. Phase Transformation Study in Nb-Mo Microalloyed Steels Using Dilatometry and EBSD Quantification. Metallurgical and Materials Transactions A. 2013; 44(8): p. 3552-3563.

5 Kasatkin, O. G.; Vinokur, B. B.; Pilyushenko, V. L. Calculation models for determining the critical points of steel. Metal Science and Heat Treatment. 1984; 26(1): p.27-31.

6 Bodnar, R. L; Hansen, S. S. Effects of Widmanstatten Ferrite on the Mechanical Properties of a 0.2 Pct C - 0.7 Pct Mn Steel. Metallurgical and Material Transactions A. 1994; 25A: p.763-773.

7 Lee, C. H.; Bhadeshia H.K.D.H.; Lee H. C. Effect of plastic deformation on the formation of acicular ferrite. Materials Science and Engineering A. 2003; 360: p.249-257. 